\title{
ANALISIS CARIOTIPICO COMPARATIVO EN NUEVE ESPECIES DEL GENERO SOLANUM L. (*).
}

DAVID TALLEDO $\left\{I_{h}\right.$ CAROLA ESCOBAR (2).

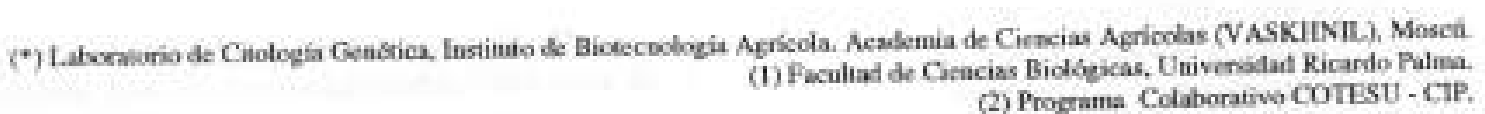

\section{RESUMEN}

El articulo presenta un analisis comparativo de los cariotipos de naeve expocies del gènero Solanum $\mathrm{L}$, ocho de ellos evidenciados por primera vez por los autores del presente trabajo. Se describea los cromosomas de cada cariotipo en función de su longitad relativa $\left(L^{\prime}\right)$, indice centromerico $\left(\mathrm{I}^{\mathrm{C}}\right)$ y otras peculiaridades individuales y se propone un sistema para elasificarlos. En base a las obscrvaciones realizsdas y a la revisión de los resultados de otres iavestigadores se sugiere el origen secundario del nuimero base $(x=12)$ de cromosomas de las especjes de genero.

\section{SUMMARY}

Tbe article presents a comparative analysis of the karyotypes of nine species of Solenum L.; eight of them are reported by the first time. This publication describes the chromosomes of each karyotype according to its relative longitude ( $\mathrm{E}^{\prime}$ ). centromeric ratio $\left(F^{*}\right)$ and other individual features, and a classification system is proposed. According to the results and bibliograpbical revision, the secondary origen of the chromosomal hase number $(x-12)$ in this species is suggested.

\section{INTRODUCCION}

Pese a los grandes éxitos en el estudio de ta citogénetica de la papa (Peloquin et, al.. 1989) el cariotipo de las especies del género Solanum aún no ha sido evidenciado, to que dificulta enormemente el fitomejocamiento anatitico (Talledo, 1991). Más auin, es imposible yuxtaponer el mapa genético de la papa. etaborado recientemente en base a la metódica deI RFLP (Gebbarult et, al _. 1989), con cromosomas somáticos concretos. Ello se debe principalmente a las peculiaridades cariológicas de la papa.

La jequeta magnitud de sus cromosomas, sa similitud morfológica y la necesidad de otilizar tratamicntos complejos para evidenciar su mortologia dificulan la identificación de los cromosomas de la papa de tal manera que muchos especialistas consideran imposible elaborar los cariotipos de las especies de este género y más aán caracterizarlos comparativamente.

La finalidad del trabajo consistió en estudiar los complementos cromosómicos, la magnitud y estructura de los cromosomas y sus peculiaridades individuales en diferentes especies silvestres del género Solanum utilizadas en el fitomejoramiento como donantes de caracteres de importancia económica.

\section{MATERIALES Y METODOS}

Se estudiaron los cariotipos de 9 especies de
Solanum: ocho silvestres diploides ( $S$. berthauliii, $S$. brevicaule, S. catorthrum, $S$. doddsii, $S$. incamayoense, S. sparsipilum, S. spegazzinii, S, vernei) y una cultivada tetraploide (S. tuberosam). Los tubérculos fueron gentilmente proporcionados por la sección de tuberosas del Instituto N.I. Vavilov de Fitotecnia.

Las láminas fueron preparadas en el Laboratorio de Citología Genética del Instituto de Biotecnologfa Agricolade VASKHINIL. (Mosco) a partir del meristemo radicular y coloreadas usando la técnica descrita por V.D. Turkov $(1974,1988)$ paraespecies de cromosomas pequeños. La estructura de los cromosomas metaf́́sicos foe estudiada por contraste de fase en un microscopio Karl Zeiss. Para el analisis cariotipico de cada especie se utilizaban no menos de 10 táminas montadas cuyos cromosomas se encontraban cou el mismo grado de espirilización. Las microfotografias de las metafases fueron tomadas con filtro naranja con película Mikrat 300. Todas las mediciones de los cromosomas fucron realizadas en fotografias en condiciones iguales. Para cada cromosoma se determinaron la longitud relativa (L') y el indice centromérico ( ${ }^{\circ}$ ). La selección de los pares de homólogos y análisis morfométrico de la estructura cromosómica fueron realizados en el Sistema de Análisis de Imágen IBAS - 2000 con el grupo de Control Genético - Ecológico de la Facultad de Agrononfa de la Academia K.A. Timiriázev de Agronomía.

Laclasificación de los cromosomas en los cariotipos se realizó segús Levan et. al., (1964). Para el procesa. miento estadistico de los resultados de las mediciones se 
observaron los criterios atilizados para los extudios cariotipicos.

\section{RESULTADOS Y DISCUSION}

En base a la $L^{r}$ y al $I^{e}$ dividimos los cromonomas de todios los cariotipos estudiados en los 6 grupos siguientes:

A: Submetacéntricos targos,

B: Submetacéntricos medianos,

C: Metacéntricos medianos.

D: Submetacéntricos pequeños.

E: Acrocéntricos con satélite,

F: Metacéntricos cortos.

En los casos cuando variaba la estructura cromosómica con escasa o nula modificación de la L', al grupo se le asignaba un subindice $\left(A_{1}, A_{2}, B_{2}+c t c\right)$.

DESCRIPCION DE LA ESPECIE SILVFSTRE DIPL.OIDE $(2 n=2 x=24)$ S. spegaseinii Bitt. serie Transaeguatorialia Buk.

En el cariotipo de csta especie encontramos los siguientes grupos de cromosomas (Fig, 1):

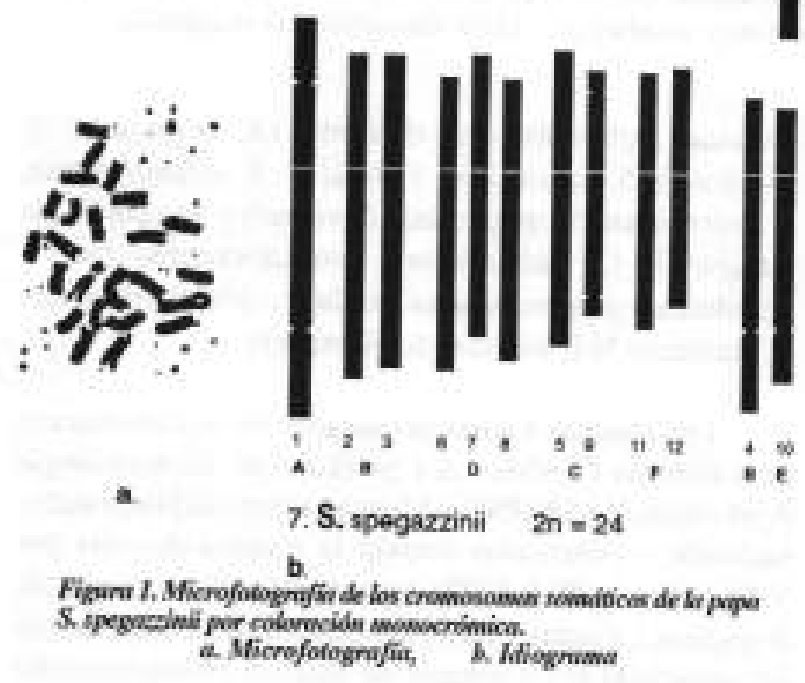

A: Primer par: Par de los cromosomas submetacéntricos más largos. Al igual que en otrak espectes del género, los cromosomas del grupo A son aproximadamente $3 \%$ más largos que los del grupo B. Presentan una constricción acéntrica en el extremo distal del brazo largo.

B: Segundo y Tercer pares: Dos pares de cromoscmas medianos, submetacéntricos más típicos que los del grupo $\mathrm{A}\left(\mathrm{I}^{\circ}=32.8\right.$ y 33,0 , respectivamente).

$\mathbf{B}_{2}$ : Cuarto par: Este par difiere poco-de los cromosomas del grupo anterior en cuanto a longitud, peroes fácilmente identificable por su morfología yaque es el único cromosoma acrocéntrico del conjunto, además del par con satélites. Astimismo, presenta una constricción acéntrica en el brazo largo. El cuario par puede ser utilizado conjuntamente con los cromosonats 12 del primer grupo como mareador.

C: Quinto y Noveno pares: L.os cromosomas de este grupo difieren por su longitud tanto de los cromosomas del grupo B como entre si $\left(\mathrm{L}^{+}=8.4\right.$ y 7.0 respectivamente), sin embargo su morfologia similar permite juntarlos en un solo grupo. Ambos pares son metacéntricos.

D: Sexto, Séptimo y Octavo pares: Grupo dc cromosomas submetacéntricos, más pequefos que los del grupo B. Los croenosomas del sếptimo par se diferencian de los de los otros 2 pares por un valor más alio de su indice centronnérico.

E: Décimo par: Par de cromosomas acrocéntricos, con satelite, heteromorfos. En el extremo proximal se evidencia una constricción secundaria que lleva un satelite. Es interesante subrayar que el satélite se observa sóloen uno de los cromosomas del par. Por otro lado, cn läminas diferentes se observahan o micro - o macro satélites, Esto permite suponer la existencia de 2 satclites y no uno en el brazo corto del décimo cromosorna, lo que seria parcialmente confirmado por observaciones análogas en otras especies (vb. en S, berthaultii). En clbrazo largo de este par de cromosomas también se evidencia una constrición acếntrica.

F: Décimo primero y Décimo segundo pares: Dos pares de cromosomas cortos. El par once es subumetacéntrico, pero no es típico; el par doce es metacéntrico. Se diferencian clarameate tanto por su tamaño $\left(L^{r}=7.3 ; L^{r}=6.7\right)$, como por los tamathos de sus brazos $\left(I^{\circ}=36.2\right.$ y 42.2 respectivameate).

\section{DESCRIPCION DE LA ESPECIE TETRAPLOIDE $(2 n=4 x=48)$ S. tuberosum L. serie Tuberosa (Rydb.) Buk.}

Al clasificar los cromosomas de esta especie fueron utilizados los mismos principios que para los croenosomas de las especies diploides.

En el cariotipo de esta especic encontramos los siguientes grupos de cromosomas (Fig. 2):

$A_{1}$ : Primer y Segundo pares : Los más grandes submetacéntricos del complemento $\mathrm{AL}^{*}=5.8: 5.7: \mathrm{I}^{*}=$ 35.8). Se observa una constricción acéntrica en el extremo del brazo largo de los cromosomas del primer par.

Be: Tercer par : Par de cromosomas metacéntricos, medianos $\left(L^{\prime}=5.2 \%, I^{\epsilon}=42.0 \%\right)$, Es muy difícil diferenciarlos de los cromosomas de los grupos A y B en base a su L', pero la ubicación del centrómero hace posible su individualización.

$B_{1}$ : Cuarto, Quinto, Sexto, Séptimo y Octavo pares: Cinco pares de cromosomas submetacéntricos, 


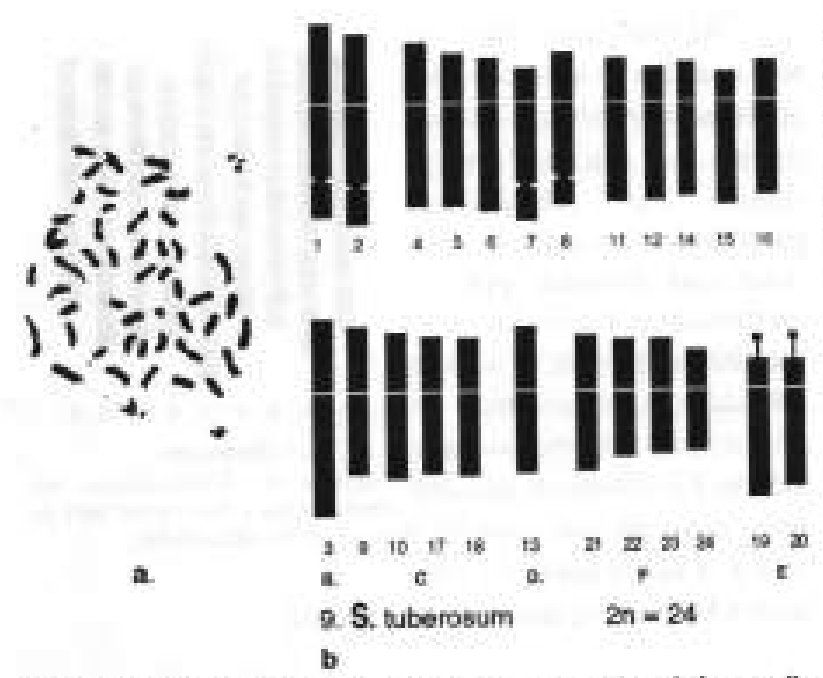

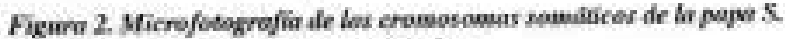
tuherosuw por colinación wanocrébeica. a. Micrefotognafix, b. Ritiogrema

medianos $\left(L^{\prime}=\right.$ de 4.8 a $4.4 \% ; I^{c}=$ de 34.5 a $\left.25.8 \%\right)$. El séptimo par de cromosomas se diferencia de los demás de este grupo por la ubicación más terminal de su centrómero $\left(\mathrm{I}^{\epsilon}=25.8 \%\right)$. En algunas láminas se evidencia una constricción acéntrica en el extremo del brazo largo en los cromosomas séptimo y octavo.

$C_{1}$ : Noveno, Décimo, Décimo séptimo y Décimo octavo pares: Cuatro pares de cromosomas metacéntricos medianos $\left(\mathrm{L}^{\prime}=\mathrm{de} 4.3 \mathrm{a} 3.7 \%\right) ;\left(\mathrm{I}^{\circ}=\mathrm{de} 43.2 \mathrm{a} 42.0 \%\right)$. Los cromosomas de los pares noveno y décimo son más largos que los de los pares décimo séptimo y décimo octavo apróximadamente en $1.0-1.5 \%$. En algunas Liminas se observa una constricción acéntrica en el brazo largo del noveno cromosoma.

Do: Décimo tercer par: Par de cromosomas metacéntricos medianos, es difícil diferenciarlos de los cromosomas del grupo anterior en base asus parámetros morfométricos. Los brazos de este ctomosoma son menos masivos que los de los cromosomas del grupo C y similares a los del grupo D.

D: Décimo primero, Décimo segundo, Décimo cuarto, Décimo quinto, Décimo sexto pares: Cinco pares de cromosomas medianos, submetacéntricos, sus longitudes relativas son aproximadamente $0.6 \%$ menores que las de los cromosomas del grupo B.

E: Décimo noveno y Vigésimo pares: Dos pares de cromosomas acrocéntricos, beteromorfos, con satélites. Su longitud relativa es de $3.7-3.6 \%$; cl fndice centromérico, 22.4-21.1\%, Tanto en el décimo novedo como en el vigésimo par los microsatélites se evidencian sólo en uno de los cromosomas, también se evidencia una constricción acéntrica en cl extremo distal de los cromosomas de ambos pares.

F: Vigésimo primero, Vigésimo segundo, Vigé. sime tercero y Vigésimo cuarto pares: Los cuatro pares más pequeflos del complemento cromosómico. En todas las láminas la ubicación del centrómero de los cromosomas vigdsimo tercero y vigésimo cuarto es más medial $\left(I^{c}=43.7 ; 45.9 \%\right)$, que en los cromosomas vigésimo primero y vigésimo segundo $\mathrm{I}^{\mathrm{e}}=39.3$; $39.4 \%)$, respectivamente.

\section{ESTUDIO DE LOS PARAMETROS MORFOLOGICOS DE LOS CROMOSOMAS DE ESPECIES SILVESTRES Y CULTIVADAS DEL. GENERO SOLANUM L.}

El análisis estadístico de los resultados en cuanto a $L^{\prime}$ e $\mathrm{I}^{\circ}$, seleccionados en base a ta $\Sigma L^{\prime}$ n y al I* de la lámina, demosuró que soló algunos cromosonas o grupos de ellos pueden ser identificados en hase a la $L^{\prime}$.

Cuando se utilizacomocriteriode individualización la $L^{r}$ y el $I^{\circ}$, en la mayoria de especies se identifica de $1 / 2$ a $2 / 3$ de los cromosomas del complemento, aunque en algunas especies ( $S$, berthaultii, $S$. cafarthrum, $S$. sparsipilum), a todos. Sin embargo, los parámetros morfométricos de los cromosomas B y D son muy parecidos, lo que dificulta su identificación. En los casos cuando entre los cromosomas de estos grupos no existen otras diferencias morfologicas es preciso utilizar métodos de coloración diferencial para su individualización.

El indice de acumacidad del experimento en la mayoría de los casos fue menor al 5\%. (Fig, 3, Fig. 4).

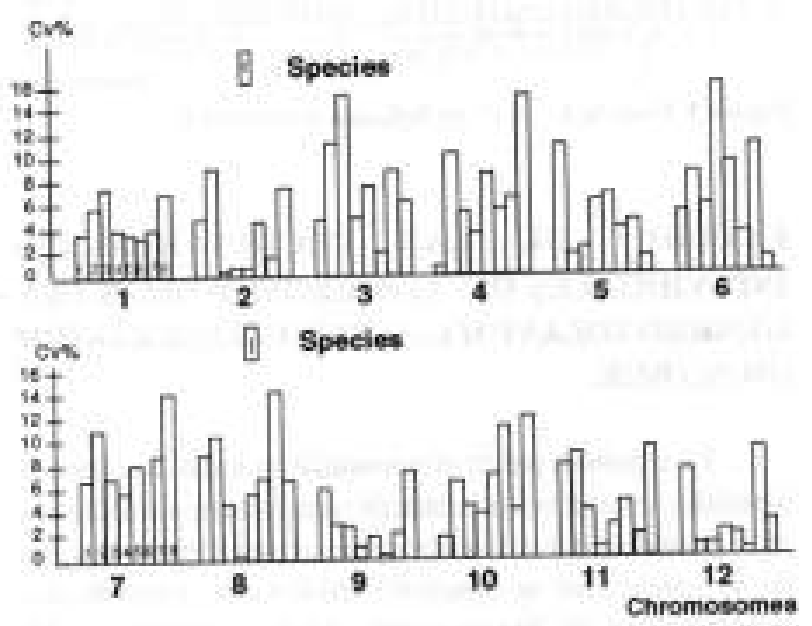

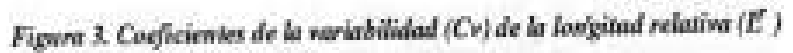
en espocies silinesines de papa.

- Nuinero de onden de las esperier (Lar nombers de las especier se preseman en el caodru I).

Los coeficientes de variahilidad de los valones de ta $L^{\prime}$ y el $I^{\mathrm{e}}$ de los cromosomas de las expecies silvestres diploides de papa en la mayoria de los casos fueron bajos (Fig. 3,4). Sólo para algunos cromosomas los coeficientes de variabilidad del $I^{\epsilon}$ fueton mayores que el $10 \%$ sin alcanzar al $20 \%$. Ello atestigua el alto grado de acuracidad de la selección de las táminas 
metafásicas con índices próximos de cspiralización. Los coeficientes de variabilidad de los parámetros morfométricos de los cromosomas de $S$, taberosum mostraron la misma conducta (Fig. 5).
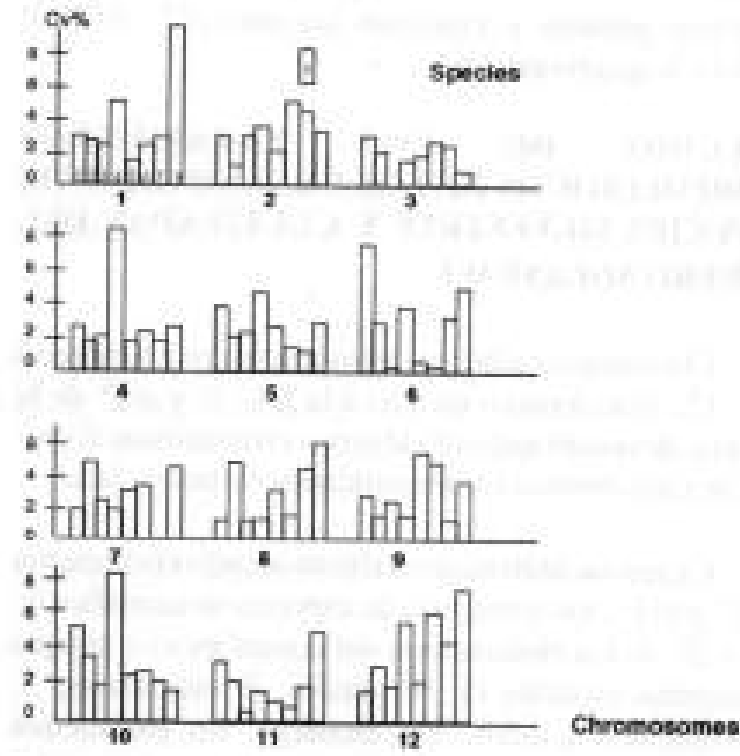

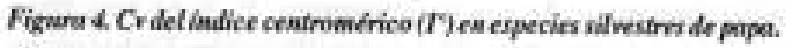

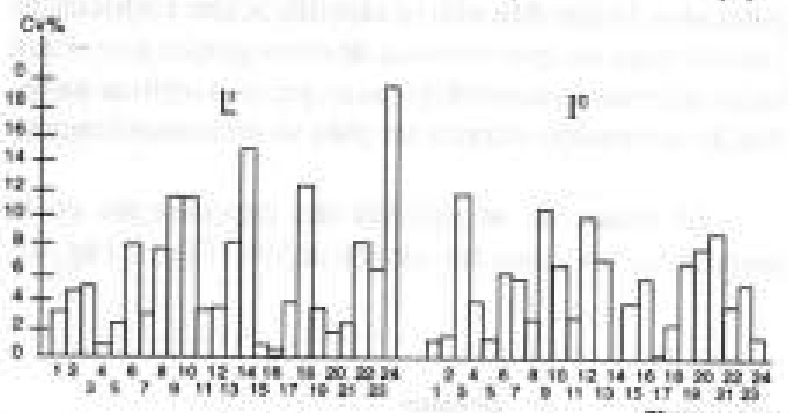

Fignera 5, Cv de la $L^{\prime}$ e $I^{e}$ en Solanum nuherosuim $L$.

\section{ESTUDIO DE LAS PECULIARIDADES INDIVIDUALES DE LOS CROMOSOMAS DEL. GENERO SOLANUM L. SECCION TUBERARIUM (DUN.) BUK.}

En el primer par de cromosomas de todas las especies điploides se evidencia claramente una constrición acéntrica en el brazo largo. En $S$ incamioyoense y en algunas láminas de $S$ spegazzinii se observa también una constricción acéntrica en el brazo corto. M.S. Ramanna y M. Wagenvoort (1976) también encontraron en el bivalente paquiténico 1 una constricción que corresponde a la constricción del brazo largo. Estos resultados alestiguan que el primer par de cromosonas somáticos corresponde al bivalenete paquindnico 1 (Fig. 6).

E par acrocéntrico de cromosomas con satélite ocupa el décimo lugar (décimo par) en nuestra clasificación, en correspondencia con su longitud relativa en la mayoria de las especies diploides estudiantas.
Al comparar estos datos con los resultados deJ análisis paquiténico encontramos que este par corres. ponde al bivalente paquiténico 2, ya que este último esta formado por los cromosomas nucleolares, [1 satelite se evidencia solo en uno de los cromosomas del par en todas las especiesestudiadas. El tamaño de los sate- Figura 6. Morfologia del

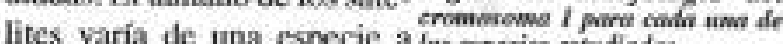
otra y a veces hasta en dife-

rentes láminas de una misma especie.

En $S$, brevicaule, $S$, incamayoense y $S$, vernei se evidenciaron macrosatelites; en $S$, catarthrum, $S$, doddsii y en $S$. sparsipilum encontramos microsatélitex. En $S$. berthoultii y $S$. spegazzinni encontramos tanto macro . como microsatelites en diferentes láminas; más aũn, en algunas láminas metaffsicas encontramos macro - y microsatelites simultíneamente, La pre. sencia de 2 satelites de diferentes tamaños en un mismo cromosoma es muy dificil de explicar sin suponer la existencia de una translocación entre 2 cromosomas con satélite (Pijnacker,

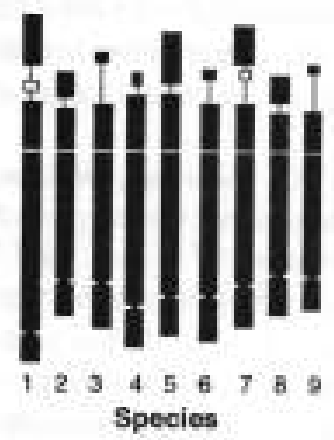

Figura 7. Morfologín de las crowosomis cow satilie parr carla uno de ins especies estubliabls Ferwerda. 1984; Pijnacker et. al., 1987) (Fig. 7).

El segundo par de cromosomas somáticos se puede reconocer por un masivo brazo largo. En todas tas especies diploides el cuarto par de cromosomas presenta una constricción acéntrica en el brazo largo; los pares guinto y noveno de $S$. brevicaule, $S$. incamayoense y $S$. vermei se caracterizan por presentar constricciones acéntricas en ambos brazos. En todas las láminas la ubicación del centrómero en el cronosoma décimo segundo es más medial que en el décimo primero, lo que facilita su identificación.

La clasificación de los cromosomas somáticos no necesariamente coincide con la clasificación de los bivalentes paquiténicos debido al acortamiento diferencial de los brazos de los cromosomas.

Pasemos a la descripciôn de los cromosomas mar. cadores de $S$. niberosum.

El primer y segundo pares del complemento tetraploide de $S$, tuberosum corresponden aparente- 
mente al primer par del complemento de las especies diploides. Al igual que en las demás especies estudiadas, en el primer par de cromosomas de $S$. tuberosum se evidencia ciaramente una constricción acéntrica en el extremo del brazo largo. En el segundo par éstaes menos evidente; el brazo corto y la región pricentromérica del brazo largo se colorean intensamente, mientras que las. regiones distales se colorean débilmente. Un cuadro similar habia sido encontrado en algunas láminas de $S$. berthaultii y S. brevicaule. En los brazos largos de los cromosomas séptimo y octavo también es posible evidenciar constricciones acéntricas. Es probable que estos pares correspondan al cuarto par del complemento cromosómico de las especies diploides.

Eatre los cromosomas del grupo C solo el noveno par presenta constricción aćntrica en uno de los brazos. Ambos pares de cromosomas con satélite presentan microsatélites.

\section{ANALISIS COMPARATIVO DE LOS CARIOTIPOS DE LAS ESPECIES DE PAPA.}

Los cariotipos de todas las especies estodiadas son bastante simétricos. Sin embargo, los componenies de esta simetria varian de una especie a otra. En las especies diploides estudiadas el número de cromosomas con los dos brazos bien desarrollados, es decir metacéntricos y submetacéntricos, varia de $83.3 \%$ en $S$. brevicaule y $S$, spegazzinii hasta $91.7 \%$, en el resto de las mismas. E nû́mero de metacénuricos varia del $50 \%$ en $S$. berthaultii i 8.3 en $S$, brevicaule. La cantidad de cromosomas metacentricos en $S$. catarthrum, $S$. incamayoense y $S$. vernei es de 41.7 , mientras que en $S$, doddsii, $S$. sparsipilan y $S$. spegazzinii es del $25 \%$.

La relación porcentual de los diferentes tipos de cromosomas para cada epecie se da en cl cuadro 1.
El estudio comparativo de las especies diploides del género Solanum nos permitió establecer los siguientes grupos morfológicos de cromosomas:

A. Primer par: Losmás grandes submetacéntricos. Su longitud relativa varía, básicamente, entre 11.0 . $11.5 \%$. Se evidencia en la mayoria de especies. El I varia de 35,2 a $36,0 \%$.

As. Primer par: Se encoentra sóloen $S$, berthaultii y $S$, catarthrum con un valor similar para la $L$ ' (11.3\%), su configuración metacéntrica $\left(\mathrm{I}^{\mathrm{c}}=38,4-40.9 \%\right)$ permite diferenciarlos de los cromosomas del grupo A de otras especies.

B. Segundo, Tercer y Cuarto pares: Los cromosomas de este grupo y sus subgrupos presentan todos los posibles pasos de metacéntricos a acrocéntricos. Los cromosomas del grupo B son submetacéntricos medianos:se evidencian en todas las especies, aunque en diferentes números. Se evidencian en su totalidad en $S$. catarthrum, $S$. incamayoense y $S$. sparsipilum. En el resto de especies diploides estudiadas este grupo no se presenta completo, ya que algunos cromosomas han sufrido determinados cambios, por lo que pasan a formar subgrupos.

B. Segundo par : Este subgrupo se encuentra sólo en $S$, berthaultii, cuyo segundo par de cromosomas es un metacéntrico tipico con un $1^{\circ}=42.3$ y una $L^{r}=9.0 \%$

$B_{1}$. Tercer par: Se encuentra solo en $S$, vernei, en esta especic cl tercer par de cromosomas es un metacéntrico típioo con un $\mathrm{I}^{\circ}=39.3$ y una $\mathrm{L}^{\prime}=9.0 \%$.

$\mathrm{B}_{2}$. Cuarto par: Este subgrupo se forma como resultado de la transformación del cuarto par subanctacéntrico en acrocéntrico $\left(I^{c}=22.3-23.9 \% ; L^{\prime}=\right.$

CUADRO I,DISTRIDUCION DE LOS CROMOSOMAS Y SUS TIPOS

\begin{tabular}{|c|c|c|c|c|c|}
\hline & $\mathrm{N}^{\circ}$ ESPECIE & \multicolumn{4}{|c|}{ TIPO DE CROMOSOMAS (\%) } \\
\hline & & $\$$ & $\mathrm{M}$ & SM & A \\
\hline 1. & $S$, berthauldii & 91.7 & 50.0 & 41.7 & 8.3 \\
\hline 2. & $S$, brevicaule & 83.3 & 8.3 & 75.0 & 16.7 \\
\hline 3. & S. catarthnum & 91.7 & 41.7 & 50.0 & 8.3 \\
\hline 4. & S. doddsii & 91.7 & 25,0 & 66.7 & 8.3 \\
\hline 5. & S. incanayoense & 91.7 & 41.7 & 50.0 & 8.3 \\
\hline 6. & S. sparsipilum & 91.7 & 25,0 & 66.7 & 8.3 \\
\hline 7. & S. spegazzinii & 833 & 25.0 & 58.3 & 16.7 \\
\hline 8. & S. vernei & 91.7 & 41.7 & 50.0 & 8.3 \\
\hline 9. & S. tuberasum & 91.7 & 41.7 & 50.0 & 8.3 \\
\hline
\end{tabular}




\section{7 - 8.9), Se presenta en $S$. brevicaule y $S$. spegazzinit.}

C. Quinto y Noveno pares : Cromosomas metacéntricos medinanos. Sc prescutan en da mayoría de especies. El indice centromérico varia de $38.0 \mathrm{a} 43,4 \%$, aunque en $S$. catarthrum el $I^{\circ}$ del quinto cromoxoma ss menor que 38.0 , mientras que el noveno cromosoma es un típico metacéntrico. La L $\mathrm{E}^{t}$ del quinto cromosoma de las especies mencionadas es aproximadiamente 1.0. $1.5 \%$ mayor que la del noveno cromosoma, exceptoen $S$. vernei que presenta ambos eromosonas casi del mismo tamano $\left(L^{\prime}=8.1 \%-7.8 \%\right.$, respectivasnente $)$. Fin al gunas especies fos cromosomas de este grupo han ewolucionado de metacéntricos a submetacéntricos, formando de esta manera $\mathrm{el}$ subgrupo $\mathrm{C}_{1}$.

C, Quinto y Noveno pares En S. brevicaule y $S$. dodsii son submetacéntricos, aunque no son tipicos y sólo en $S$. sparsipilam tanto el quinto como el noveno pares son submetacéntricos.

D. Sexto, Séptimo y Octave paresi Cromosomas submetacéntricos medianos, aunque más pequeños que los del grupo B y sus subgrupos. Se encuentran en iodas las especies, aunque en diferente nùmero; la longitud relativa varia de 8.3 a $7.6 \%$; el indice centromérico, de 32.0 a $27.8 \%$. El séptimo par de cromosomas de $S$. berthaultit, S. brevicaule, S. catanthnam y S, spegazzinii presenta mayores valores del indice centromérico que los demás del grupo. En algunas especies este grupo estí formado solo por los pares 6 y 8 , ya que el sćptimo par ha pasado a formar un subgrupo,

D, + Séptimo par: Cromosoma mediuno, típien metacéntrico $\left(1^{\circ}=41,2-40,0 \%\right)$. Se cncucntraen $S$, dhodvii, $S$. incamayoense y S. sparsipilum.

E. Décimo par: Cromosonat acrocéntrico $\left(\mathrm{I}^{\mathrm{F}}=\right.$ $24.0-21.1 \%)$, de variada lengitud en las diferentes especies $\left(L^{\prime}=9.2-7,1 \%\right)$. El hrazo corto de uno de los cromosomas del par presenta una constricción secundaria que lleva a un satelite. Se evidencia en todas las especies. El tamaño del satélite varia en las dif́erentes especies.

F. Décimo primeroy Décimosegundo pares: Doc pares de cromosomas pequefios, metacéntricos en La mayorade especies, excepto $S$, brevicaule. S. sparsipilam y $S$. spegozzini, en las cuales el decuno primer par es suhmetacéntrico. Sin embargo, solo en $S$, brevicaule el décimn primer par es un submetacéntrico tipico. En todas las especies el décimo segundo par es un metacéntrico más típico que el décimo primer par. liaridades:

Es imprescindible recakar dos interesantes pecu-

1. Al conparar los complementos haploides de 8 especies silvestres de papa encontramos que se repiten alguans cromosomas iguales o muy parecidos. Esto se refiere principalmente a los grupos B y D, asi como a los cromosomas de loc grupos C y $F$, cuya magnitud se diferencia ligeramente aunque su morfologfa es similar

2. Los grapos $A, B, C$ y D presentun los subgrupos $A_{6}: B_{0}, B, y=B_{2} ; C_{r} \cdot D$, en función de las difertintes vanaciones del íddice centromérico. En el gnupo B es posible observar la evolución de los cromosomas melacéntricos hacia submetacéntricos $(B$, y $B, \rightarrow \rightarrow \rightarrow B)$ $y$ de los suhmetactuntricus a los acrocéntricos $\left(B \rightarrow \rightarrow \rightarrow B_{2}\right)$. L.a evolución gradual de los cromosmanas de un tipo hacia otro permile suponer que tas variaciones de sa estroctura presentan importancia filogenética para tas especies del género Solanumı L. (Fig. 8).

Nuestros resultados demuesiran que los complementos cromoxomicos de las especies estudiadas presentaa los 6 grupors de cromosomas. Sin embargo, en algunas especies estos cromosomas han sufrido de. terminadas variaciones diando connoresultado la formacióa de

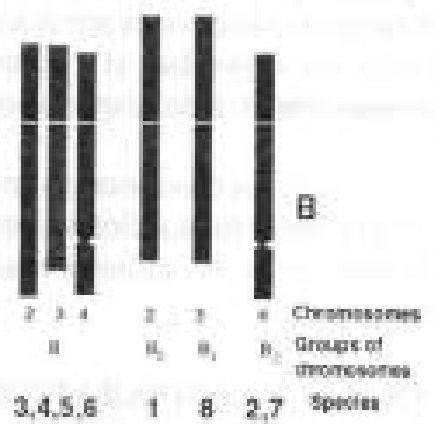

$\begin{array}{lllll}3,4,5,6 & 1 & 8 & 2,7 & \text { spovien }\end{array}$

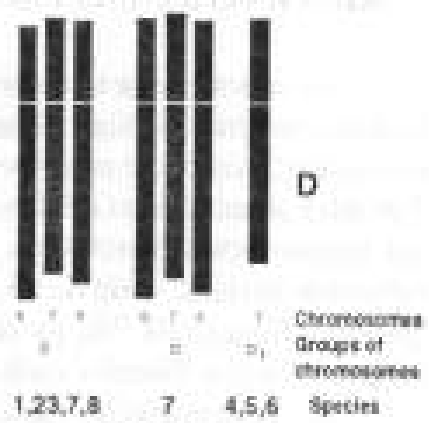

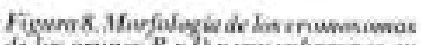

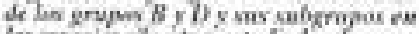

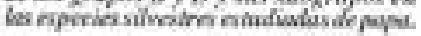
subgrupos. Ello se confirma, además, por el hecho que ca los complementos que presentan los crumoscmas modificados no es posible evidenciar su forma originai.

Cuando comparamos ei caristipo de S, nuberosum con tes cariotipos de las especies silvestres, podemes presumir que el genomade $S$. fuberosim se ha formado como resultado de la integración de los genomas de dos especies diplö̈des diferentes, Esto seria confirmado por cl dohle número de cromosamas en todos los grupes dẹ cariotipo de $S$, laherosam y por la presencia de cronosonas matcadores en los grupos B y D

Nucstros resultados atestsguan el importante papel que han descmpenado tas aberriciones cromosómicts cul laevolución de las expecies de papa. Loscrumosomas de morfologia igual o muy similar en cl complenento taploide evidenciades en nuesuro trahajo; la semejanza de la coloración diferencial entre los cromosomas de hos grupos B y D así como al interior de los grupos $\mathrm{C}$ y F en una forma momohaploide de $S$, tuberesum $\mathrm{L}$. (Pijnacker et. al.. 1984, 1987, 1989); al igual que fenómenos curacterísticos de las formas de origen tetrapioide, observados 
y descritos en diferentes especies diploides de papa; husos multipolares (Ramanna, 1979), gametos sin reducir (Mok et. al., 1975: Mok, Peloquin, 1975 b; Peloquin. 1983), autosindesis (Emme, 1936; Gilles, 1955), lo mismo que el rol evidente de la poliploidia en la ewolución de la papa sugieren fuertemente el origen secundario del nutmero base de cromosomas de las especies del género Solanun. Probablemente el número hase de cromosmass de papa $x=12$ apareció como resultado de la potiploidizacióa del número base primario $x=6$, con la ulterior diferenciación de los cromosomas involucradks, lo que podría explicar la eompleja comblucta de estas especies durante los procesos citogenéticos.

\section{CONCLUSIONES}

1. Se ha estandarizado una metódica para el conteo de cromosomas y el estudio de su morfología con la utilización de inhibidores miqcicos a hajas lemperaturas pxsitivas y maceración enzimática. Fillo permite la ideatificación de pares de homólogos.

2. Se han elaborado los cariotipos de nueve especies del género Solanum: incluyendo las especies diploides silvestaes $S$. berthaulfii, $S$. brevicate, $S$. catarthrum, $S$. doddsii, S. incamavoense, S. sparsipilum, $S$. spegazzinii. $S$ vernei y la especie uctraploide cultivada $S$. fuberosum, en base a métodos de análisis cuantitativo de los cromosomas.

3. Los cromosomas $1,5,9,10,11$ y 12 así comno los grupos 2-4 y $6-8$ de los cariotipos de toxlis las especies silvestres estudiadas han sido identificados en hase at su morfología y parámetros morfométricos.

4. Se han determinado los cromosomas marcideres o grupo de ellos para todas las especies estadiadas en base a coloración monoctómica considerando las peculiaridades morfologicas de los cromosomas y sus narámetros morfométricos.

5. Entre la mayoría de las especies estudiadas bemos encontrado similitud morfologica de sus

\section{REFERENCIAS BIBL.IOGRAFICAS}

EMME, E.K. 1936. Hibridos triploidesde Solonum antipoviczi resistentes a la fitóftors. Botanicheskii Zhumal. 5:4kh, in russian).

GEBHARDT, C; RITTER, E.; DERENER, T: SCHACHTSCHABEL, $U$; WAL.KEIMEK, R, UHKING, H.ANDSAIAMINI, $\boldsymbol{F}$. 19R9. RII.P analysisand linkaces mapping in Solanum tuberosiom. Theoe. $\mathrm{MPOL}$. Genet. 78 : $67-75$

GIL.LES, A. 1955. Recherches cytogenetiques sur les Solamum (Section Tuberarium 1. 1. Nounhres chronsisinaiques ef associations meiotiques. Cellule - Louvain $57 ; 7.71$. cromosounas, con excepción de los cromosomas marcadores de cadit especie concreta.

6. Se establecieron diferencias significativas entre los parámetros morfológicos de los cromosomas marcadores de los cariotipos de las especies de papa estudiatdas, así como entre los cromosomas de cada cariotipo. En los cariotipos de las especies de papa estudiadas foe evidenciado el polimorfismo cromosómico.

7. Fue evidenciado el heteromorfismo del par de cromosomas con satćlite, asi como el polimorfismo de este par entre las diferentes especies..

8. Fue establecida unaalta simetriade los cariotipos de las especies diploides silvestres y de la especie tetraploide cultivada; más aún, todas las especies presentarom mo menos de 8, 3\% decromosomas delcariotip con anbos brazos bien desarrollados.

9. Fin los complemenios haploides fuerma encontridos grupos de cromosomas (B y D) de idéntica morfología, astnque de diferente tamaño. El mismo fenómeno se oherva entre los cromoxomas de los grupos $C$ y F de la mayoria de especies.

10. Nuestros resultados y los de otros investigadores permiten suponer el origen secundario det ab́mero base de cromosomas de papa $(x=12)$ mediante la poliploidización y ultcrior diferenciación de los genomios involucrados. EI origen tetraploside de los complementos diploides podría explicar no solo has divergancias al deterninar el aúmero hase de cromosomas de las expecies de Solanum I.. sección Tuberaritum (Dun,) Buk.. simo también la compleja conducta de estas expecies durante los procesos citogenćlicos.

11. Nuesuros resultados demucsiran que La variatoilidial senotipica de las estructuras cromosomicas ha jugado un papel importante con laevolución de lakespecics đel género Solansun sección Tuberanizm.

L.EVAN, A.; FIEDGA, K.; SADBERG, K, 1964, Nomenelature tor centomeric position of chromosomes. Heredilas. 52 . $201 \cdot 220$

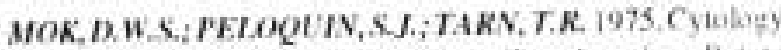

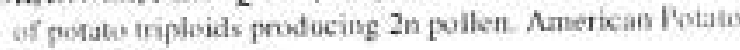
Joumall. 52:171-174.

MOK. I.W.S.; NELOQEIN, S.J. 1975 h. The inheritane of ibree meshanisms of diplandroid i $2 n-$ pollen f formation

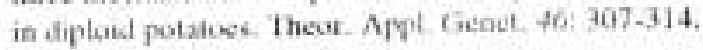

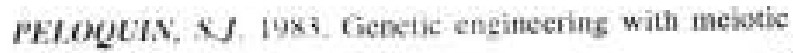


mutants. Pollen: Biological implications for Plant Breeding. N.Y. 151-155.

PELOQUIN, S.J.; JANSKY,S.H.; YERK, G.L. 1989. Potate cytogenetics and germplasm. American Potato Journal. 66: 629.638 .

PIJNACKER, L P.; FERWERDA, M. 1984, Giemsa C. banding of potato chromosomes. Canadian Journal of Geneties and Cytology. 26: 415-419.

PIJNACKER, L P.;FERWERDA, M.; PUITE, K.J.; ROEST, S. 1987. Elimination of Solanum phareja nocleolar chromosome in $S$, tabereswmx $S$. phureja somatic hybrids. Theor. Appl. Genet. 73: 878-882.

PIJNACKER, L P,; FERWERDA, M.; PUITE, K.J.; SCHAART, J.G. 1989, Chromosome elimination and mutation in tetraploid somatic bybrids of Solansm tuberosum and Solanwm phureja. Plant Cell Reports. 82.85.

RAMANNA, M.S. 1979. A re-exumination of the mechanisms of $2 \mathrm{n}$-gamete formation in potato and its implication for breeding. Euphytics, 28: 537-561.

RAMANNA, M.S. WA GENVOORT, M. 1976, Identifieation of the trisomic series in diploid Solanum zwberosum L. group Tuberosiem. 1. Chromosome identification. Euphylica. 25: 233-240.

TALLEDO, D. 1991, Estudio cariotipico comparativo de especies silvestres del género Solanum, utilizadas en el fitomejoramiento como donantes de esracteres de importancia económica. Ph. D. Thesis, Moscow. 152 pp. (Fn ruso).

TURKOV, V.D.; SHELEPINA, G.A. 1974, Caracterización cariotipica de la papa cultivada (Solanum tuberasum). Ponencias de VASKHNIL. 11: 18-19. (En ruso).

TURKOV, V.D.; GUZHOV, YU. L; SHELEPINA, G.A.; KISHMARIA, Y. SH.; KOMETIANI, D.G. 1988. Los estudios cromosómicos ea vegetales y su utilización en los problemas del fitomejoramiesto, la ingeniería celular y el monitoreo genético, M., 1988, 64 p. (En ruso). 